\title{
Pasteurella species peritoneal dialysis-associated peritonitis: Household pets as a risk factor
}

\author{
Philippe Guillaume Poliquin MD FRCPC ${ }^{1}$, Philippe Lagacé-Wiens MD FRCPC ${ }^{2,3}$, Mauro Verrelli MD FRCPC ${ }^{4}$, \\ David W Allen $\mathrm{MD}^{5}$, John M Embil MD FRCPC ${ }^{2,6}$
}

PG Poliquin, P Lagacé-Wiens, M Verrelli, DW Allen, JM Embil. Pasteurella species peritoneal dialysis-associated peritonitis: Household pets as a risk factor. Can J Infect Dis Med Microbiol 2015;26(1):52-55.

BACKGROUND: Pasteurella species are Gram-negative coccobacilli that are a part of the normal oropharyngeal flora of numerous domestic animals. They have been recognized as a rare but significant cause of peritonitis in patients undergoing peritoneal dialysis (PD). A consensus about management strategies for $\mathrm{PD}$-associated peritonitis caused by Pasteurella species currently does not exist.

METHODS: The microbiological database serving the Manitoba Renal Program was searched from 1997 to 2013 for cases of Pasteurella species PD-associated peritonitis, and charts were reviewed. PubMed was searched for case reports and data were abstracted.

RESULTS: Seven new local cases and 30 previously reported cases were analyzed. This infection is clinically similar to other forms of PD peritonitis, with household pet exposure appearing to be the strongest risk factor. Cats are the most commonly implicated pet. Direct contact between the pet and the equipment was commonly reported (25 of 37 patients) but was not necessary for infection to develop. The mean duration of treatment was 15 days. Complication rates were low, with only $11 \%$ of patients requiring PD catheter removal. There was no mortality reported.

CONCLUSION: Pasteurella species are a rare cause of PD-associated peritonitis that can be successfully treated with a two-week course of intraperitoneal antibiotics with a high likelihood of catheter salvage.

Key Words: Cat; Pasteurella multocida; Pasteurella species; Peritoneal dialysis; Peritonitis

Dasteurella multocida is a Gram-negative coccobacillus first identified in 1878 in diseased birds (1). Since then, $P$ multocida has become associated primarily with skin and soft tissue infections following animal bites. The organism is known to colonize the upper respiratory tract of $90 \%$ of cats and $66 \%$ of dogs (2). Contamination with P multocida may result in a wide range of infections including pneumonia, endocarditis and meningitis (1).

Among more unusual sites, $P$ multocida has been found to cause peritonitis in individuals undergoing peritoneal dialysis (PD) for renal replacement (3). Patients undergoing PD consider it to be a convenient alternative to hemodialysis that is associated with a reduced impact on their quality of life (4). One of the major drawbacks of PD, however, is the risk for peritonitis due to frequent manipulation of the catheter and PD equipment (5). Despite improvement in infection

\section{La péritonite associée à la dialyse péritonéale causée par des espèces de Pasteurella : les animaux domestiques sont un facteur de risque}

HISTORIQUE : Les espèces de Pasteurella sont des coccobacilles à Gram négatif qui font partie de la flore oropharyngée normale de nombreux animaux domestiques. Ils constituent une cause de péritonite rare, mais importante, chez les patients sous dialyse péritonéale (DP). Il n'y a pas de consensus quant aux stratégies de prise en charge de la péritonite associée à la dialyse péritonéale causée par les espèces de Pasteurella.

MÉTHODOLOGIE : Les chercheurs ont exploré la base de données microbiologiques du Programme de lutte contre la maladie du rein du Manitoba de 1997 à 2013 pour déceler les cas de péritonite associée à la DP causée par les espèces de Pasteurella et ont examiné leur dossier. Ils ont fouillé PubMed pour trouver des rapports de cas et en ont extrait des données.

RÉSULTATS : Les chercheurs ont analysé sept nouveaux cas locaux et 30 anciens cas. Cette infection est similaire sur le plan clinique à d'autres formes de péritonite associée à la DP, dont le principal facteur de risque semble être l'exposition à un animal domestique. Les chats sont les plus en cause. On signale souvent un contact direct entre l'animal et le matériel (25 patients sur 37), mais il n'est pas nécessaire pour provoquer l'infection. Le traitement durait en moyenne 15 jours. Le taux de complications était faible, puisque seulement $11 \%$ des patients ont dû faire retirer leur cathéter de DP. Aucun décès n'a été signalé.

CONCLUSION : Les espèces de Pasteurella sont de rares causes de péritonite associée à la DP qu'on peut soigner par un traitement de deux semaines aux antibiotiques intrapéritonéaux. Ce traitement s'associe à une forte probabilité de sauvegarder le cathéter.

rates due to better equipment and a focus on hand hygiene, an event rate of 0.5 episodes/patient/year is average (6).

Over the past 15 years, eight cases of $P$ multocida PD peritonitis have been observed within the Manitoba Renal Program (Winnipeg, Manitoba), which currently provides care to 280 PD patients. In the present article, we briefly describe seven of these cases; the eighth case was previously reported in the Canadian Journal of Infectious Diseases $\mathcal{E}^{2}$ Medical Microbiology (7) due to its unique features as a polymicrobial zoonosis. In addition, we reviewed and analyzed the available published case reports of Pasteurella species PD-associated peritonitis $(2,3,7-30)$. While the burden of such infections is small in comparison with the usual microbiology, both the prevalence of chronic renal disease (31) and pet ownership are increasing (32). As a result, we anticipate that there will be an increase in cases of PD peritonitis

${ }^{1}$ Section of Paediatric Infectious Diseases, Department of Paediatrics and Child Health; ${ }^{2}$ Department of Medical Microbiology and Infectious

Diseases, University of Manitoba; ${ }^{3}$ Department of Clinical Microbiology, St Boniface General Hospital; ${ }^{4}$ Section of Nephrology, Department of Internal Medicine, University of Manitoba, and Manitoba Renal Program; ${ }^{5}$ Section of Cardiology; ${ }^{6}$ Section of Infectious Diseases, Department of Internal Medicine, University of Manitoba, Winnipeg, Manitoba

Correspondence: Dr John M Embil, Infection Prevention and Control Unit, Health Sciences Centre, MS673, 820 Sherbrook Street, Winnipeg, Manitoba R3A 1R9. Telephone 204-787-4654, fax 204-787-2989, e-mail jembil@hsc.mb.ca 
caused by Pasteurella species. Collectively, analysis of the 37 available cases enabled the creation of meaningful recommendations to guide future therapy.

\section{METHODS}

\section{Identification of local cases}

The electronic microbiology laboratory database that serves the Manitoba Renal Program was searched for P multocida or Pasteurella species isolated from peritoneal fluid from January 1, 1997 to June 30, 2013. Patient charts were abstracted for the following: age, sex, comorbidities, PD history, animal exposure and symptoms. Laboratory data, including white blood cell (WBC) counts from peripheral blood and peritoneal fluid as well as Gram stain and culture results, were collected.

\section{Review of published reports}

The PubMed database was searched from 1966 to June 1, 2013 using the MeSH terms ("Pasteurella"[Mesh] OR "Pasteurella pneumotropica"[Mesh] OR "Pasteurella multocida"[Mesh] OR "Pasteurella Infections"[Mesh]) AND ("Peritoneal Dialysis"[Mesh] OR "Peritoneal Dialysis, Continuous Ambulatory"[Mesh] OR "Peritonitis"[Mesh]). This search returned 64 citations. Cases describing a PD-associated episode of peritonitis caused by Pasteurella species were included. Bibliographies were reviewed for additional cases. Individual reports were abstracted for the same information as the local cases.

\section{Local case series}

All of the cases included in the present series ultimately yielded $P$ multocida from culture of the PD fluid obtained at the time of presentation. None of these local patients experienced a relapse of PD peritonitis caused by Pasteurella species. All patients were initially treated using the PD program's standing protocol for the empirical treatment of PD peritonitis - specifically, once-daily intraperitoneal (IP) cefazolin (or vancomycin for beta-lactam allergic patients) and tobramycin. Table 1 demonstrates dosing guidelines used at the authors' institution.

Case 1: A 28-year-old woman with a history of tetralogy of Fallot, hypertension and congenital solitary kidney developed end-stage renal disease (ESRD) managed by continuous ambulatory peritoneal dialysis (CAPD). She had been on CAPD for one month and presented with a $5 \mathrm{~h}$ history of severe abdominal pain and chills. Her cat had chewed on her dialysate line earlier that day. She was started on IP cefazolin and tobramycin for $48 \mathrm{~h}$. Treatment was tailored to IP ceftazidime for an additional 12 days, with clinical improvement within $24 \mathrm{~h}$.

Case 2: A 37-year-old man who had been on CAPD for 15 months for ESRD secondary to diabetic nephropathy sought medical attention for a one-day history of abdominal pain, fever and chills. His cat had bitten the dialysate line the day before, although a puncture was not observed. He was started on IP cefazolin and tobramycin, and experienced prompt recovery. The tobramycin was discontinued after five days and he completed 14 total days of IP cefazolin.

Case 3: A 41-year-old man who had been on CAPD for ESRD secondary to diabetic and hypertensive nephropathy for the past 18 months sought medical attention for a $4 \mathrm{~h}$ history of abdominal pain, fever, chills, nausea, vomiting and diarrhea. The patient noted that his dialysate fluid had been cloudy that morning. He was the owner of three cats that were allowed into the PD area, and reported that one may have bitten the tubing. He demonstrated rapid improvement after initiation of IP cefazolin and tobramycin. Tobramycin was discontinued on day 5 and he completed 14 days of IP cefazolin.

Case 4: A 51-year-old woman who had been on CAPD for the past seven months for ESRD secondary to hypertension sought medical attention for a $5 \mathrm{~h}$ history of abdominal pain, fever, nausea and vomiting. She indicated that her dialysate fluid was cloudy during her most recent session. Although she owned a cat, she reported that it was not permitted within the PD area. The patient was treated with three days of IP cefazolin and tobramycin, with marked improvement within $24 \mathrm{~h}$
TABLE 1

Intraperitoneal antibiotic dosing

\begin{tabular}{ll}
\hline Antibiotic & Dose \\
\hline Cefazolin & $1.5 \mathrm{~g}$ intraperitoneal once daily if $\geq 50 \mathrm{~kg}$ \\
& $1 \mathrm{~g}$ intraperitoneal once daily if $<50 \mathrm{~kg}$ \\
Ceftazidime & $1.5 \mathrm{~g}$ intraperitoneal once daily \\
Vancomycin & If no residual renal function: \\
& $2 \mathrm{~g}$ intraperitoneal every 7 days if $\geq 50 \mathrm{~kg}$ \\
& $1 \mathrm{~g}$ intraperitoneal every 7 days if $<50 \mathrm{~kg}$ \\
& If patient has residual renal function with urine output \\
& $>100 \mathrm{~mL} /$ day: \\
& Same empirical doses as above; however, dosing is \\
& adjusted after vancomycin level is obtained four to five \\
days after initial dose
\end{tabular}

of therapy. IP antibiotics were discontinued and patient completed 14 days of amoxicillin-clavulanic acid orally.

Case 5: A 37-year-old woman who had been on CAPD for 11 years for ESRD secondary to chronic interstitial nephropathy sought medical attention for a $10 \mathrm{~h}$ history of abdominal pain, chills and diarrhea. She had regular contact with a domestic cat but not in the vicinity of her PD equipment. She was treated with IP cefazolin and ceftazidime for five days but had ongoing cloudy dialysate and abdominal pain. The PD catheter was removed, and she completed her therapy with three days of ceftriaxone and one week of oral amoxicillin.

Case 6: A 59-year-old woman who had been on CAPD for the past three years for ESRD secondary to diabetic nephropathy sought medical attention for a four-day history of nausea and vomiting and a one-day history of abdominal pain. The woman had regular contact with a domestic cat in her home, but no documented contact with PD equipment or tubing. She received a five-day course of IP cefazolin and tobramycin followed by IP ceftazidime for 14 days with complete recovery.

Case 7: A 69-year-old woman with an underlying history of diabetes mellitus, hypertension, thalassemia and diabetic nephropathy was started on CAPD. She presented three days later with cloudy dialysate and subjective fever. She reported abdominal pain with her latest fluid exchange. Concern was raised that her cat may have bitten the cycler line. She was discharged from the emergency department with reassurance but was asked to return because P multocida was recovered in the PD fluid culture. She was successfully treated as an outpatient with two days of IP cefazolin and tobramycin followed by seven days of IP ceftazidime and seven days of oral amoxicillin-clavulanic acid.

\section{RESULTS}

The local database search revealed the seven cases described above. An additional published case from our centre (7) was added to 29 published cases identified using the search strategy described above. This resulted in a total of 37 cases for analysis. The vast majority (33 of 37) of cases were secondary to $P$ multocida, with one each of $P$ aerogenes, $P$ canis, $P$ dagmatis and $P$ pneumotropica accounting for the remainder.

\section{Demographics}

The mean $( \pm$ SD) patient age was $44.4 \pm 17.6$ years (range eight to 75 years), with a male-to-female ratio of $1: 1$. There were three pediatric patients. Cases were heterogeneous regarding the underlying reason for renal failure, including both congenital as well as acquired causes. PD modality varied, with $43 \%$ using continuous cycling PD, $41 \%$ using CAPD and $16 \%$ being unspecified. The mean length of time on PD was 24.1 months, with a wide range (three days to 11 years).

\section{Clinical and laboratory features at presentation}

The median time to presentation was $18.5 \mathrm{~h}$ (range $1 \mathrm{~h}$ to $168 \mathrm{~h}$ ). Information on clinical presentation was available for 34 of 37 patients. Abdominal pain was most commonly reported (91\%). Other signs and 
TABLE 2

\section{Management approaches for patients with Pasteurella} peritoneal dialysis-associated peritonitis

\begin{tabular}{lc}
\hline Initial management & $\begin{array}{c}\text { Proportion of patients } \\
\text { receiving specified } \\
\text { treatment }\end{array}$ \\
Intraperitoneal antibiotics only & $27 / 37(73)$ \\
Intravenous antibiotics only & $4 / 37(11)$ \\
Combination intravenous/intraperitoneal/oral & $3 / 37(8)$ \\
No therapy & $2 / 37(5)$ \\
Definitive therapy & \\
Intraperitoneal antibiotics only & $19 / 34(56)$ \\
Intravenous antibiotics only & $6 / 34(18)$ \\
Combination intraperitoneal/oral & $3 / 34(9)$ \\
Oral antibiotics only & $6 / 34(18)$ \\
Duration of therapy, days, mean \pm SD (range) & $15.2 \pm 4.1(7-24)$ \\
Duration of therapy, days, median & 14 \\
\hline
\end{tabular}

Data presented as $n /$ total $n$ (\%) unless otherwise indicated

symptoms included: nausea and vomiting $(65 \%)$, cloudy dialysate fluid (50\%), fever (38\%), chills (29\%) and diarrhea (12\%). No patients were asymptomatic. A tunnel site infection was diagnosed in one of 37 patients.

The peripheral WBC count was available for 26 of 36 patients, with an average WBC count of $10.8 \times 10^{9} / \mathrm{L}\left(3.7 \times 10^{9} / \mathrm{L}\right.$ to $\left.20.7 \times 10^{9} / \mathrm{L}\right)$. Numerically, the WBC was abnormal in only $54 \%$ of cases (as per the reported normal range for individual institutions). The peritoneal fluid cell count was universally abnormal, with an average of $6621 \times 10^{6} / \mathrm{L}$ (range $210 \times 10^{6} / \mathrm{L}$ to $25,879 \times 10^{6} / \mathrm{L}$; normal $<100 \times 10^{6} / \mathrm{L}$ ). The Gram stain was not reliably helpful, demonstrating Gram-negative rods in only six of 33 cases. Blood cultures were not routinely drawn; two patients had $P$ multocida bacteremia, one asymptomatically and one with a shock-like syndrome.

\section{Animal type and degree of contact}

Cats were the most commonly implicated animal, accounting for $83 \%$ (31 of 37) of cases. Exposure to animals other than cats occurred conclusively in four cases. This appeared to be more common in the few pediatric patients (two of three nonfeline exposure) and resulted in non-P multocida infections. Direct contact between the animal and the equipment was documented in 25 of 37 cases, of which 10 of 25 confirmed a puncture of the line or fluid bags. An additional 10 cases reported no contact between the animal and PD equipment or treatment area. There was no association between type of exposure and PD modality $(\mathrm{P}=0.304)$. There was a significant difference in time from symptom onset to presentation between patients with a bite or puncture of the PD catheter tubing compared with patients with nonspecific contact ( $15 \mathrm{~h}$ versus $44 \mathrm{~h} ; \mathrm{P}=0.04$ ).

\section{Management and outcomes}

The approach to management of these infections was highly variable within the reported literature in terms of both route of delivery and length of therapy (Table 2). Outcomes were generally favourable. Two patients experienced a septic shock-like syndrome requiring admission to the intensive care unit. One patient experienced a recurrence four weeks after discontinuation of therapy. The PD catheter was removed in $11 \%$ (four of 37 ) of patients. There was no discernible association between a particular therapeutic regimen and a poor outcome. No mortality was reported.

\section{DISCUSSION}

Since the first case of P multocida peritonitis was described in 1987, this organism has become recognized as an infrequent but clinically significant cause of PD-associated peritonitis (3). The seven cases from our centre represent the largest series reported to date. Together with the existing 30 published reports, important trends emerge $(2,3,7-30)$.
Overall, patients with Pasteurella species PD-associated peritonitis have a very similar symptom constellation to other forms of infectious peritonitis. When our patients were compared with patients from a recent case series of $\mathrm{PD}$-associated peritonitis (5), rates of abdominal pain $(91 \%$ versus $88 \%)$, nausea and vomiting ( $65 \%$ versus $51 \%)$ and fever (38\% versus 29\%) were similar. Observation of a cloudy effluent was less common in infections caused by Pasteurella species (50\%) compared with other organisms (84\%). This may be due to the relatively rapid median time to presentation for care of $18.5 \mathrm{~h}$. Given that cases occurred in PD recipients with both lengthy (11 years) and limited (third day of PD) experience, it is evident that ongoing education regarding the risk of $\mathrm{PD}$-associated peritonitis in the presence of household pets is critical.

Exposure to a colonized animal is a prerequisite of infection; however, the degree of contact necessary for infection appeared to be limited. Direct contact between the animal and the equipment was documented in 25 of 37 cases, of which 10 of 25 confirmed a puncture of the line or fluid bags. More intriguing were the 10 cases that reported only casual contact between the owner and the animal. While a surreptitious contact event cannot be excluded, the observation that animal breeders can acquire oropharyngeal colonization with Pasteurella species raises the possibility that self-inoculation from the patient's own flora may underlie some of these infections (33). Furthermore, it has been demonstrated using pulsed-field electrophoresis that Pasteurella species oropharyngeal colonization with the same organism can occur in both patient and pet (24).

The most recent recommendations of the International Society for Peritoneal Dialysis recommend a three-week course of antimicrobial therapy for Gram-negative peritonitis (34). Based on previously published reports and our case series, it appears that, when compared with infection caused by other Gram-negative microorganisms, patients with PD-associated peritonitis caused by Pasteurella species experienced fewer recurrences and catheter loss events. The vast majority of these patients were successfully treated with a 14-day course of antimicrobial therapy, primarily delivered intraperitoneally. The one recurrence occurred in a patient treated for three weeks (11). Shorter courses of therapy (as short as seven days) have provided equivalent outcomes but the data are insufficient to allow definitive conclusions about shorter duration of therapy. Catheter loss was uncommon (four of 37). This compared favourably with a previously reported rate of $23 \%$ in non-Enterobacteriaceae Gramnegative-induced peritonitis (5). As such, a 14-day course of IP antibiotic therapy aimed at catheter salvage appears to be warranted for PD-associated peritonitis caused by Pasteurella species. On average, substantial clinical improvement occurred within $48 \mathrm{~h}$ to $72 \mathrm{~h}$ of initiation of therapy. The lack of mortality is reassuring, especially given that $P$ multocida bacteremia typically carries a $30 \%$ mortality rate (22).

Given the heterogeneity of antibiotic choices, it is difficult to draw definitive conclusions about optimal antimicrobial choice. In general, however, penicillin- or ampicillin-based regimens are preferred for non- $\beta$-lactamase-producing isolates (1). Third-generation cephalosporins are believed to have equivalent activity to penicillin and ampicillin. Oral fluoroquinolone monotherapy was successfully used in five of 33 patients. Aminoglycoside IP monotherapy was used in three patients $(3,11,17)$, one of whom (11) experienced the only recurrence following three weeks of therapy. This event, together with the unreliable activity of aminoglycosides against Pasteurella species described in the literature (35), suggests that aminoglycoside monotherapy in Pasteurella species peritonitis should be avoided.

There are several limitations inherent to retrospective review of isolated case reports. Those most pertinent to the present report include: patient recall bias, specifically with regard to animal exposure; inconsistent reporting/documentation of risk factors (such as type and extent of animal exposure) leading to infection; publication bias; differences in practice patterns within and between centres; and reliable laboratory identification of rare microorganisms. Recognizing the limits of this type of study, we believe that sufficient points of commonality have emerged to allow these trends to be reported. 


\section{CONCLUSION}

The present case series and review of published reports is the largest and most complete to date, and provides a clear picture of PD-associated peritonitis caused by Pasteurella species. This infection is indistinguishable from other forms of PD peritonitis except for a tendency toward rapid $(<24 \mathrm{~h}$ of symptoms) presentation for care. These organisms should be suspected as an etiological agent if there is a pet at home, even if direct contact between the pet and the equipment was not observed. Moreover, a history of a puncturing animal bite to PD tubing or fluid bags should raise the index of suspicion. Gram stain and WBC counts from both peripheral blood and peritoneal fluid are not helpful in distinguishing Pasteurella species from other infectious agents causing PD-associated peritonitis. This infection can occur in both novel and experienced PD users. As a result, PD patients should be regularly asked about pet ownership. Periodic reminders of infection control procedures should be stressed in routine follow-up appointments of PD patients with household pets.

\section{REFERENCES}

1. Zurlo JJ. Pasteurella species. In: Mandell GL, Bennett JE, Dolin R, editors. Mandell, Douglas, and Bennett's Principles and Practice of Infectious Diseases, Seventh Edition. Philadelphia: Elsevier, 2010:2939-42.

2. Sillery J, Heargreaves J, Kuznia C, Abbe C. Pasteurella multocida peritonitis: Another risk of animal-assisted therapy. Infect Control Hosp Epidemiol 2004;25:5-6.

3. Paul RV, Rostand G. Cat bite peritonitis: Pasteurella multocida peritonitis following feline contamination of peritoneal dialysis tubing. Am J Kid Dis 1987;10:318-9.

4. Juergensen E, Wuerth D, Finkelstein SH, Juergensen PH, Bekui A, Finkelstein FO. Hemodialysis and peritoneal dialysis: Patients' assessment of their satisfaction with therapy and the impact of the therapy on their lives. Clin J Am Soc Nephrol 2006;1:1191-6.

5. Oliveira LG, Luengo J, Caramori JC, Montelli AC, Cunha Mde L, Barretti P. Peritonitis in recent years: Clinical findings and predictors of treatment response of 170 episodes at a single Brazilian center. Int Urol Nephrol 2012;44:1529-37.

6. Troidle L, Finkelstein F. Treatment and outcome of CPD-associated peritonitis. Ann Clin Microbiol Antimicrob 2006;5:6.

7. Al-fifi Y, Sathianathan C, Murray B-L, Alfa M. Pets are 'risky business' for patients undergoing continuous ambulatory peritoneal dialysis. Can J Infect Dis Med Microbiol 2013;24:e96-8.

8. Antony SJ, Oglesby KA. Peritonitis associated with Pasteurella multocida in peritoneal dialysis patients - case report and review of the literature. Clin Nephrol 2007;1:52-6.

9. Campos A, Taylor JH, Campbell M. Hamster bite peritonitis: Pasteurella pneumotropica peritonitis in a dialysis patient. Pediatr Nephrol 2000;15:31-2.

10. Castellan I, Marín JP, Gallego S, et al. Pasteurella canis peritonitis in a peritoneal dialysis patient. Perit Dial Int 2011;31:503-4.

11. Cooke FJ, Kodjo A, Clutterbuck EJ, Bamford KB. A case of Pasteurella multocida peritoneal dialysis-associated peritonitis and review of the literature. Int J Infect Dis 2004;8:171-4.

12. Frankel AH, Cassidy MJD. Pasteurella multocida peritonitis in CAPD: Beware of the cats. Perit Dial Int 1991;11:84-5.

13. Freeman AF, Zheng XT, Lane JC, Shulman ST. Pasteurella aerogenes hamster bite peritonitis. Pediatr Infect Dis J 2004;23:368-70.

14. Iwashima K, Tsujimoto Y, Tabata T, et al. Two case reports of Pasteurella peritonitis in peritoneal dialysis. Nihon Toseki Igakkai Zasshi 2008;41:213-18.

15. Joh J, Padmanabhan R, Bastani B. Pasteurella multocida peritonitis following cat bite of peritoneal dialysis tubing. Am J Nephrol 1998;18:258.

16. Kanaan N, Gavage P, Janssens M, Avesani V, Gigi J, Goffin E. Pasteurella multocida in peritoneal dialysis: A rare cause of peritonitis associated with exposure to domestic cats. Acta Clinica Belgica 2002;57:254-6.

17. Loghman-Adham M. Pasteurella multocida peritonitis in patients underdoing peritoneal dialysis. Pediatr Nephrol 1997;11:353-4.
The suggestion in the International Society for Peritoneal Dialysis guideline of empirical Gram-positive and Gram-negative therapy for $\mathrm{PD}$-associated peritonitis is appropriate even if Pasteurella species are suspected (34). If Pasteurella species are recovered from PD fluid culture, we suggest a 14-day course of IP therapy guided by antimicrobial susceptibilities; aminoglycoside monotherapy should be avoided. Antibiotic-based PD catheter salvage therapy is an appropriate goal given that this approach was successful in $90 \%$ of published cases.

DISCLOSURES: The authors have no conflicts of interest to declare.

ACKNOWLEDGEMENTS: There were no study sponsors or funding sources.

ETHICS APPROVAL: Ethics approval was not required for this study.
18. Mackay K, Brown L, Hudson F. Pasteurella multocida peritonitis in peritoneal dialysis patients: Beware of the cat. Perit Dial Int 1997;17:608-10.

19. Malik A, al Aly Z, Mailey KS, Bastani B. Pasteurella multocida peritoneal dialysis-associated peritonitis: A report of two cases and review of the literature. J Nephrol 2005;18:791-3.

20. Mat O, Moenens F, Beauwens R, Rossi C, Muniz-Martinez M-C. Indolent Pasteurella multocida peritonitis in a CCPD patient. 25 years of "cat-bite peritonitis": A review. Perit Dial Int 2005;25:88-90.

21. Mugambi SM, Ullian ME. Bacteremia, sepsis, and peritonitis with Pasteurella multocida in a peritoneal dialysis patient. Perit Dial Int 2010;30:381-3.

22. Musio F, Tiu A. Pasteurella multocida peritonitis in peritoneal dialysis. Clin Nephrol 1998;1998:258-61.

23. Rondon-Berrios H, Trevejo-Nunez GJ. Pets or pest: Peritoneal dialysis-related peritonitis due to Pasteurella multocida. J Microbiol Immunol Infect 2010;43:155-8.

24. Satomura A, Yanai M, Fujita T, et al. Peritonitis associated with Pasteurella multocida: Molecular evidence of zoonotic etiology. Ther Apher Dial 2010;14:373-6.

25. Uribarri J, Bottone EJ, London RD. Pasteurella multocida peritonitis: Are peritoneal dialysis patients on cyclers at increased risk? Perit Dial Int 1996;16:648.

26. van Langenhove G, Daelemans R, Zachee P, Lins RL. Pasteurella multocida as a rare cause of peritonitis in peritoneal dialysis. Nephron 2000;85:282-4.

27. Wallet F, Toure F, Devalckenaere A, Pagniez D, Courcol RJ. Molecular identification of Pasteurella dagmatis peritonitis in a patient undergoing peritoneal dialysis. J Clin Microbiol 2000;38:4681-2.

28. Weiss GA, Panesar M. Pasteurella multocida peritonitis with bacteremia on initiation of peritoneal dialysis. Perit Dial Int 2012;32:363-4.

29. Hamai K, Imai H, Ohtani H, et al. Repeated cat-associated peritonitis in a patient on automated noctornal intermittent peritoneal dialysis. Clin Exp Nephrol 1999;1:51-61.

30. Makin AJ, Cartwright KA, Banks RA. Keeping the cat out of the bag: A hazard in continuous ambulatory peritoneal dialysis. BMJ 1991;303:1610-1.

31. Arora P, Vasa P, Brenner D, et al. Prevalence estimates of chronic kidney disease in Canada: Results of a nationally representative survey. CMAJ 2013;185:E417-23.

32. Perrin T. The business of urban animals survey: The facts and statistics on companion animals in Canada. Can Vet J 2009;50:48-52.

33. Donnio P, LeGoff C, Avril J, Pouedras P, Gras-Rouzet S. Pasteurella multocida: Oropharyngeal carriage and antibody response in breeders. Vet Res 1994;25:8-15.

34. Li P, Szeto C, Piraino B, et al. Peritoneal dialysis-related infections recommendations: 2010 Update. Perit Dial Int 2010;30:393-423.

35. Stevens D, Higbee J, Oberhoffer T, Everett E. Antibiotics susceptibilities of human isolates of Pasteurella multocida. Antimicrob Agents Chemother 1979;16:322-4. 


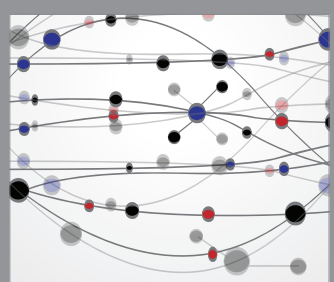

The Scientific World Journal
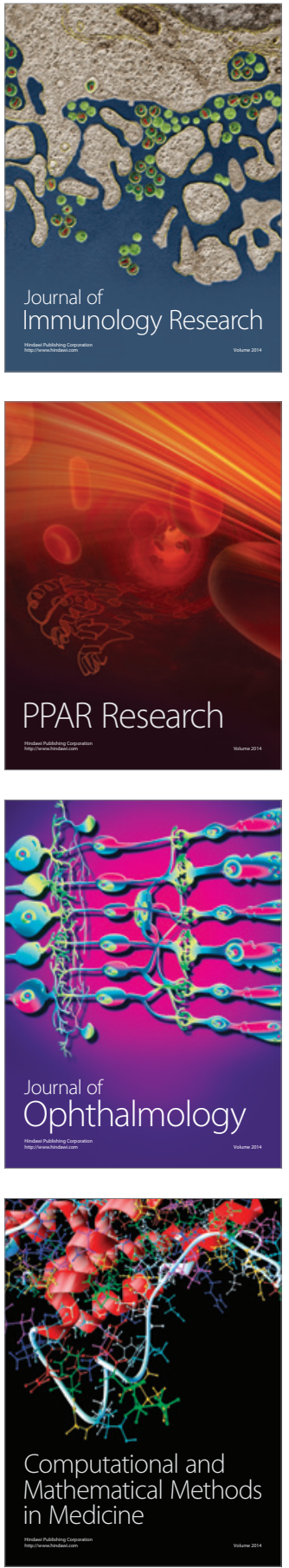

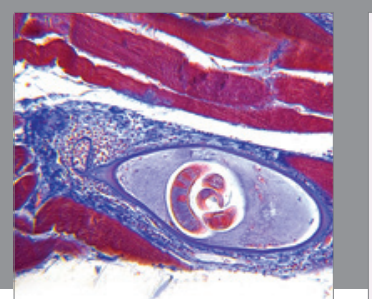

Gastroenterology Research and Practice

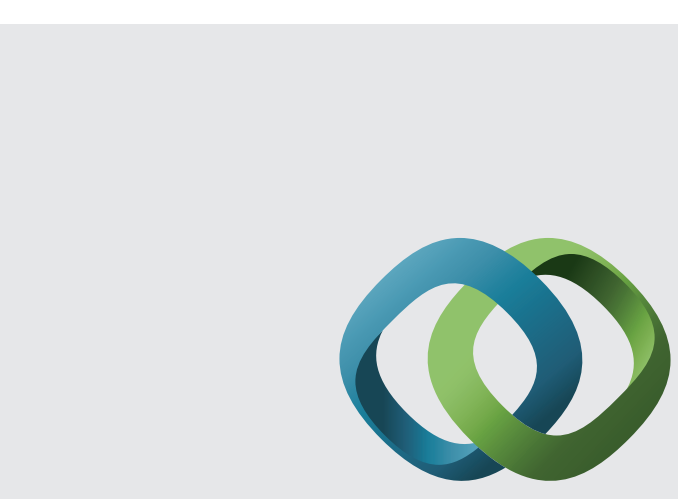

\section{Hindawi}

Submit your manuscripts at

http://www.hindawi.com
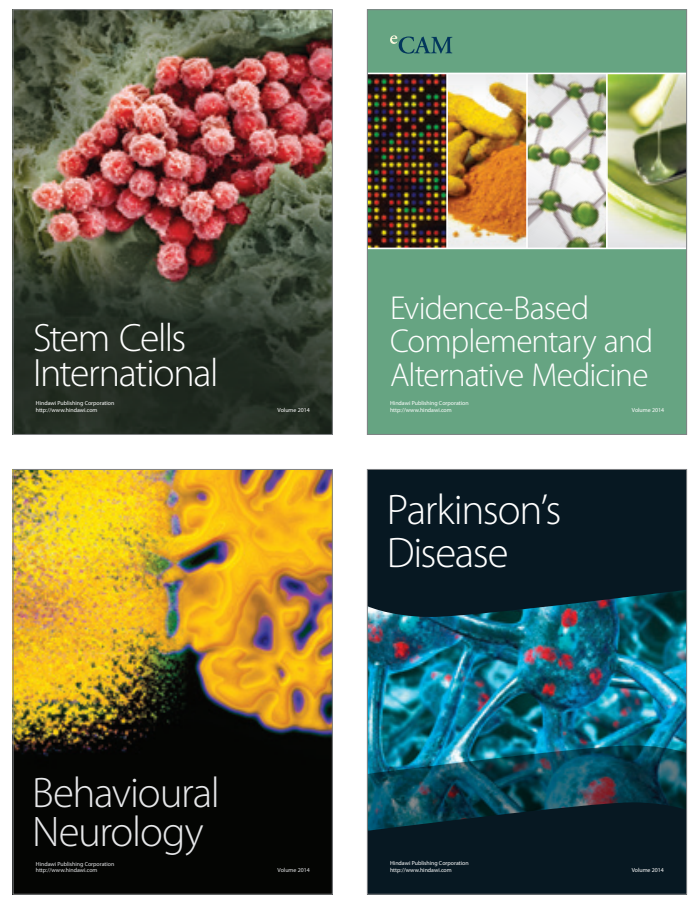
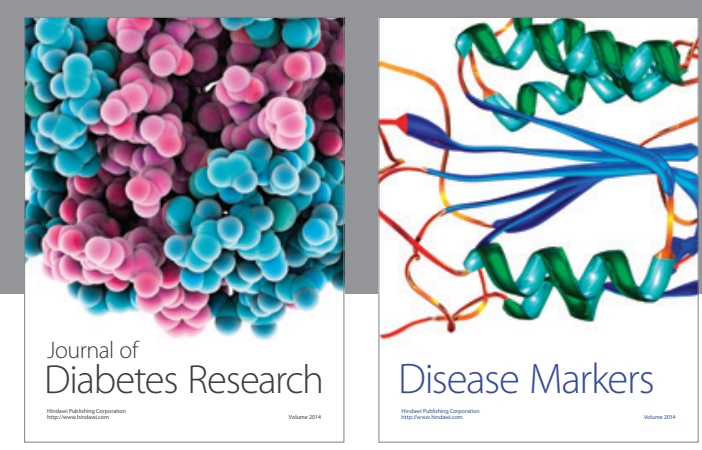

Disease Markers
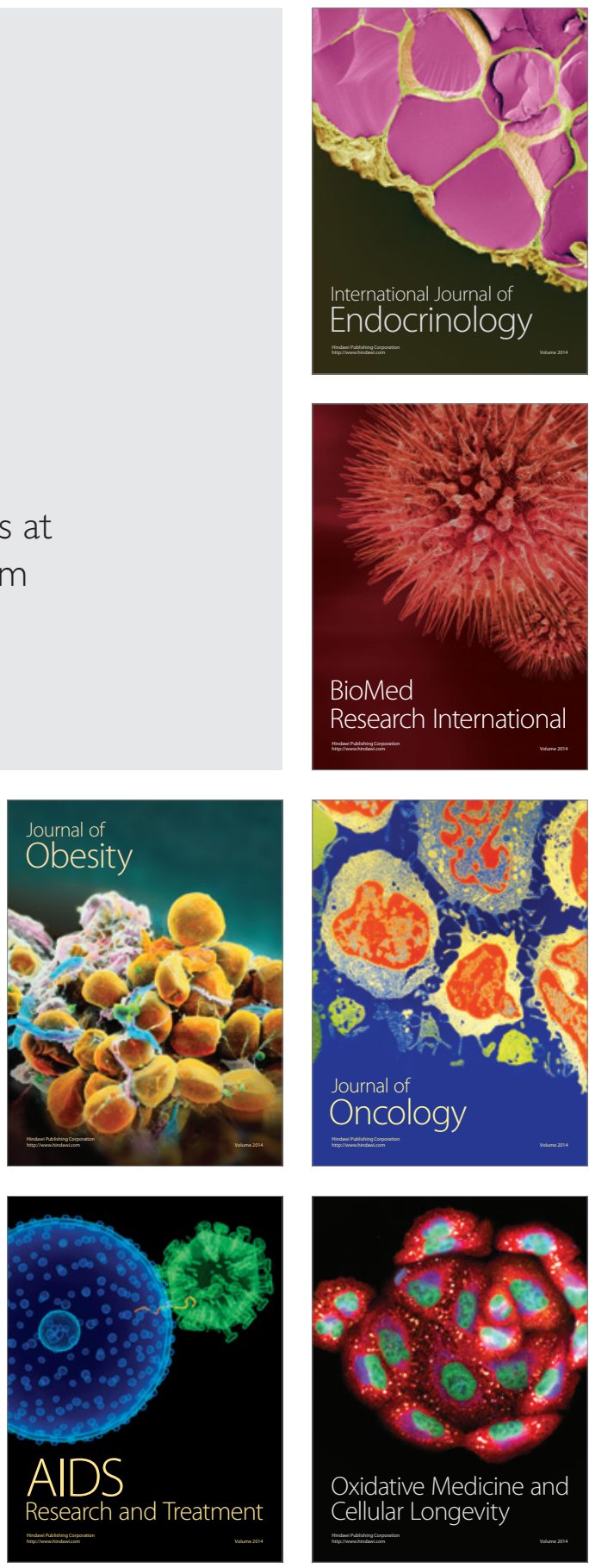